\title{
Excellency of Converse Scalping Forehead Flap for Reconstruction of External Nasal Soft-Tissue Defects
}

https://doi.org/10.47210/bjohns.2021.v29i1.456

\section{Gaurab Ranjan Chaudhuri ${ }^{1}$}

$\underline{\text { Introduction }}$

\section{ABSTRACT}

Reconstruction of external nasal defects poses a challenge for the plastic surgeons. The scalping forehead flap, first described by Converse in 1942 is a versatile technique for subtotal and total external nasal reconstruction. The flap is extremely reliable with robust vascularity and provides excellent colour and texture match for the external nose with minimal donor-site morbidity. Materials and Methods

In this series, eleven patients with external nasal soft-tissue defects (post traumatic and post excisional) were reconstructed with scalping forehead flap; among them four presented with congenital naevus, three with post-traumatic external nasal defect, two presented with basal cell carcinoma, and two with arterio-venous malformation. The age of the patients ranged between 24 and 67 years; eight male and three female patients were included in this study. Among them three had comorbidities like diabetes mellitus and/ hypertension.

$\underline{\text { Results }}$

All the flaps survived nicely. I didn't face any complication in any of my patients. The external nasal defects were reconstructed with scalping forehead flap in two stages with an interval of three weeks in between.

Conclusion

The Converse scalping forehead flap is an excellent option in the armamentarium of plastic surgeons for subtotal and total nasal reconstruction because of its reliability, vascularity, and simplicity even in the era of microvascular free tissue transfer. $\underline{\text { Kevwords }}$

Surgical Flaps; Forehead; Angiosome; Scalping Forehead Flap; Rhinoplasty

$\mathrm{R}$ econstruction of large external nasal defects remains a challenge for the plastic surgeons. For a satisfactory functional and aesthetic outcome, the flap must have a robust vascularity, needs to match the colour and texture of the nose, should be pliable and have an inconspicuous donor site.

The scalping forehead flap represents one of the best techniques for reconstruction of subtotal and total external nasal defects. The flap is supple enough to be folded; required to reconstruct the lobular portion of the nose, provides perfect colour and texture match for the nasal skin and caters adequate dimension of soft tissue for complete reconstruction of external nose.

The scalping forehead flap was described by Jean Marquise Converse of the New York University School of Medicine, in 1942. ${ }^{1}$ It is an axial pattern flap with abundant vascularity from superficial temporal artery as well as from the branches of the ophthalmic artery, the supraorbital and supratrochlear arteries. There is a rich vascular anastomoses between the vessels making the flap robust and reliable.

A pedicle of hair-bearing scalp is used to move soft tissue from lateral forehead onto the nose for reconstruction of large defects. The flap is well

1 - Department of Plastic Surgery, RG Kar Medical College, Kolkata

\section{Corresponding author:}

Dr Gaurab Ranjan Chaudhuri

email: chaudhurigaurab@gmail.com 


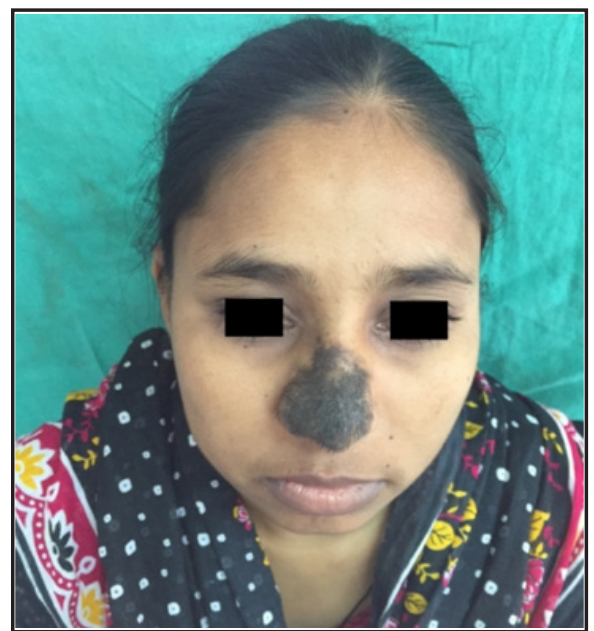

Fig. 1A. Congenital hairy naevus involving external nose

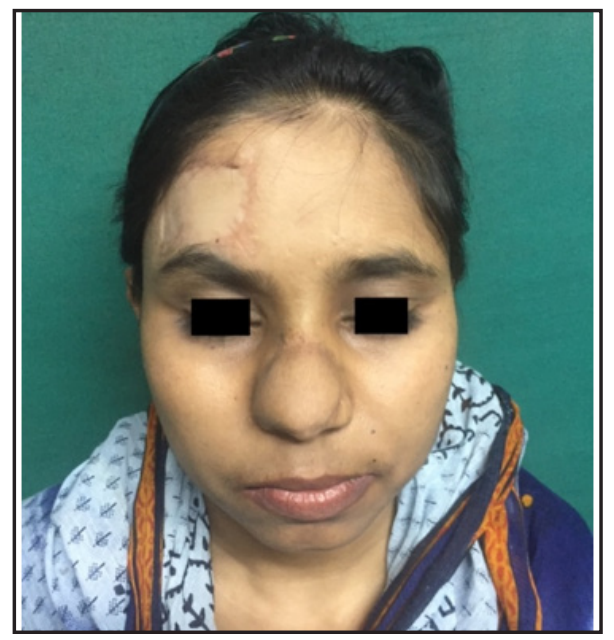

Fig. 1D. Postoperative appearance after six months

vascularised, reliable, easy to perform and results aesthetically pleasing donor site.

\section{Materials and Methods}

In this series I have reconstructed external nasal softtissue defects (post traumatic and post excisional) with Converse scalping forehead flap in eleven patients; among them four presented with congenital naevus, three with post-traumatic external nasal defect, two presented with basal cell carcinoma, and two with arterio-venous malformation.

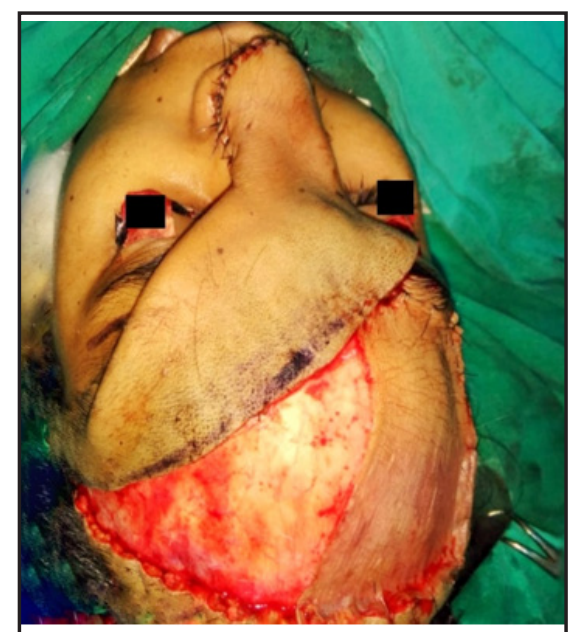

Fig. 1C. Flap inset done over the recipient site and flap donor site covered with skin graft

The patients were admitted from the outpatient department. The study was done between 2014 and June 2019 in the Department of Plastic Surgery. The age of the patients ranged between 24 and 67 years; eight male and three female patients were included in this study. Among them three had comorbidities like diabetes mellitus and/ hypertension.

The procedures followed were in accordance with the protocols of the ethical committee on human experimentation and the Helsinki Declaration of 1975, as revised in 2000. Informed consent was obtained from the patients included in this study.

\section{Surgical Technique:}

After complete excision of the lesions or freshening of the post-traumatic wounds, a pattern of the nasal defect was made with a lint and transferred over the lateral aspect of the forehead. The flap design was made at least $1 \mathrm{~cm}$ larger than the defect to allow for flap retraction. The flap was raised superficial to muscle over the frontalis and rest of the flap was dissected in the subgaleal plane leaving the underlying pericranium intact. (Fig. 1) Motor innervation to the frontalis muscle is via the seventh cranial nerve through the undersurface of the muscle. Therefore one has to remain superficial to the frontalis muscle while raising the flap to preserve this innervation. ${ }^{2}$ 


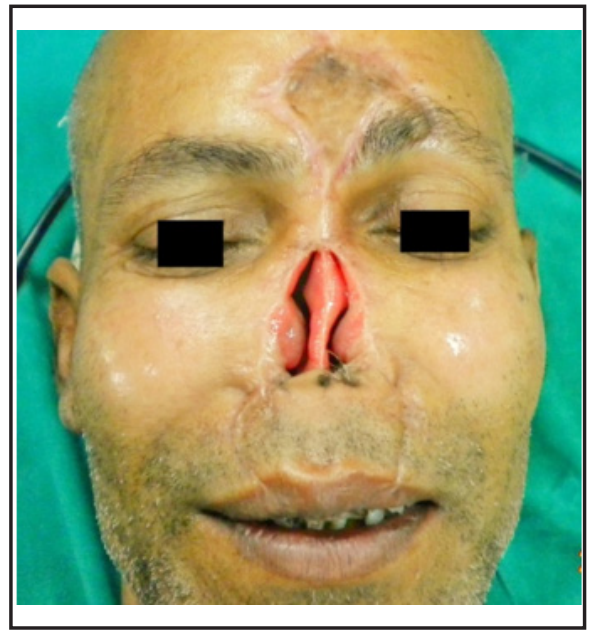

Fig. 2A. Post traumatic total amputation of nose

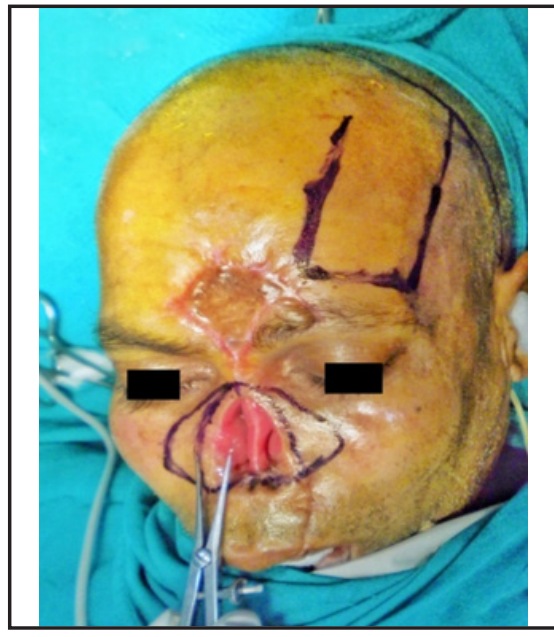

Fig. 2B. Planning of flaps for lining and cover

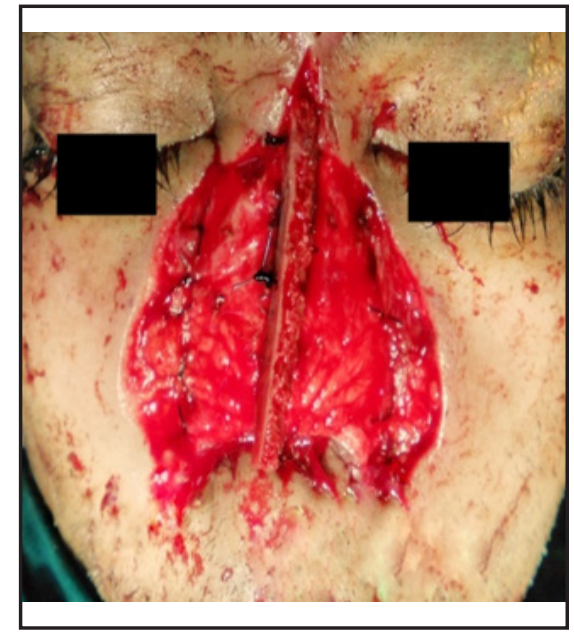

Fig. 2C. Inner lining reconstructed with defect based bilateral paranasal flaps and cantilever iliac crest bone graft for structural support
After completion of flap incisions, the incision was extended from the lateral border of the flap posteriorly with a coronal incision to the apex of the contralateral auricle, incorporating the contralateral superficial temporal vessels. The flap was then harvested and transferred to the external nasal defect without tension. The lateral forehead defect over the frontalis muscle was covered with a full-thickness skin graft. (Fig. 2) The scalp defect was covered with non-adherent dressing.

In all cases I have performed the second-stage procedure after 3 weeks. The flap pedicle was divided

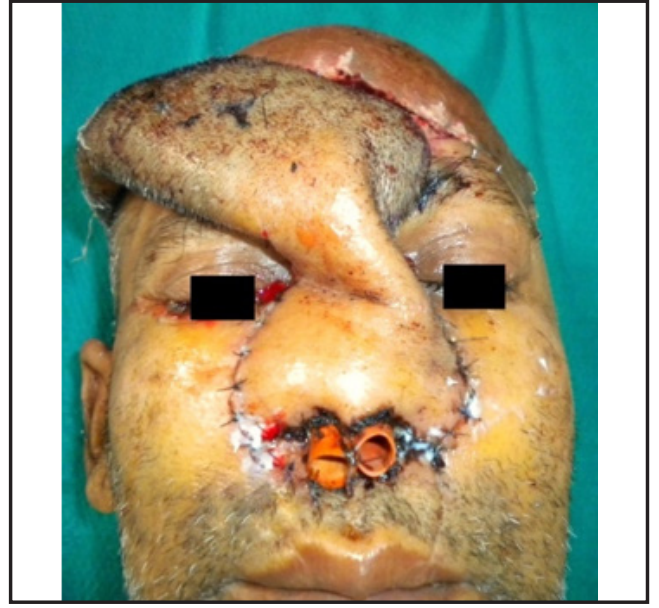

Fig. 2D. Outer cover reconstructed with scalping forehead flap and final inset was done; the proximal part of the flap was returned to its original donor site over the scalp and the forehead.

Additional support for the dorsum of the nose or internal lining of the nose can be reconstructed in conjunction with the Converse flap and may include septal composite grafts, bone graft or cartilage graft. ${ }^{3}$ I used cantilever bone graft harvested from iliac crest in one of my patients who presented with post-traumatic total amputation of nose. (Fig. 3)

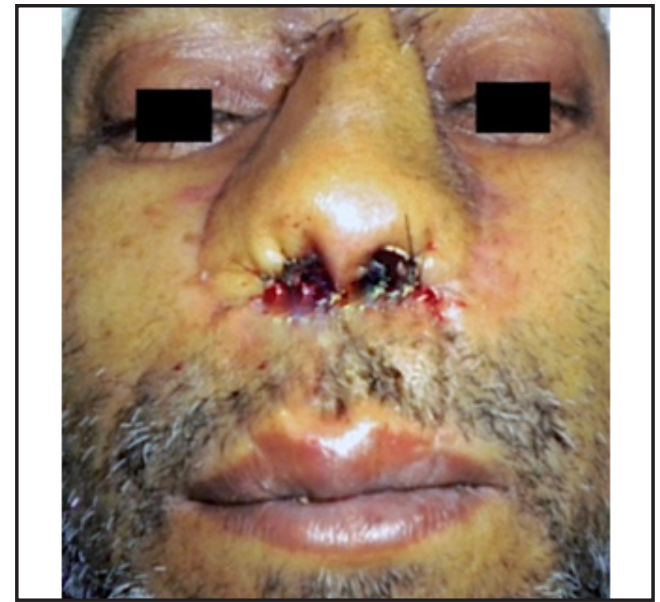

Fig. 2E. After flap division and final inset 


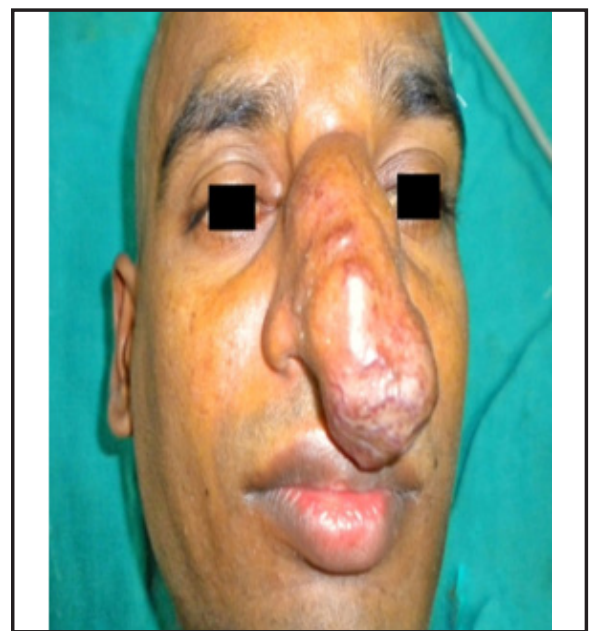

Fig. 3A. Arteriovenous malformation involving external nose

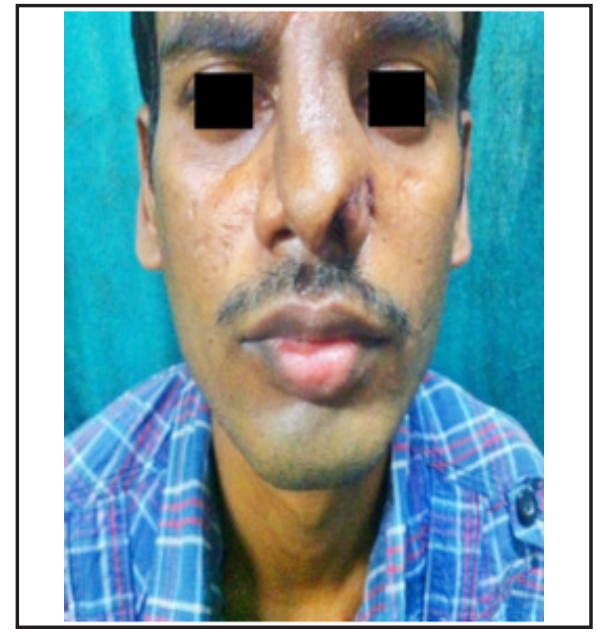

Fig. 3D. Postoperative appearance after three months

When required late revision surgery can be performed, such as flap defatting, reconstruction of the nasal framework and restoration of nostril symmetry to improve the aesthetic appearance of the nose.

\section{Discussion}

The use of tissue expanders in the forehead to create 'extra skin' for reconstruction of large nasal defects has been well described, ${ }^{4}$ but is not favoured on occasions because of high cost, frequent hospital visits for inflation of the expanders, long duration of treatment and needs

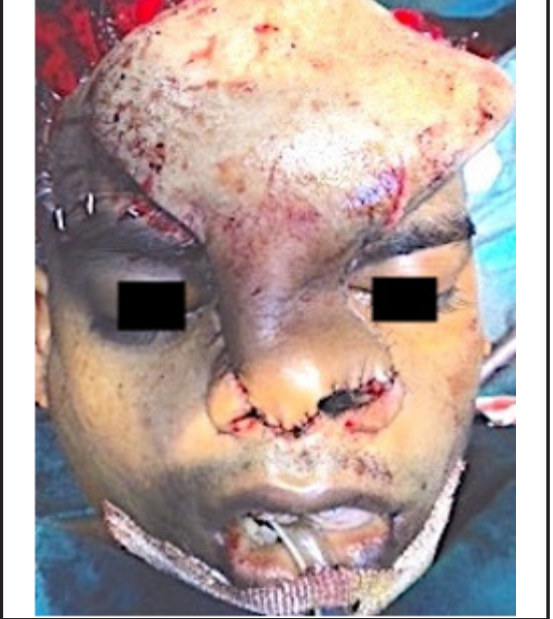

Fig. 3C. External nasal defect reconstructed with scalping forehead flap and flap donor site covered with skin graft

multiple stages for reconstruction. The scalping flap is an excellent alternative in regards to reconstruction with 'like' tissue, reliability, simplicity and pleasing functional and aesthetic outcome.

The use of forehead tissue for nasal reconstruction dates back to the India in 600BC. ${ }^{5}$ Reconstructive techniques described in literature for external nasal defects include the midline forehead flap, the paramedian forehead flap, the Gilles 'up-and-down' flap and the McGregor transverse forehead flap. ${ }^{6,7}$ The main drawbacks of those techniques are difficult to reconstruct subtotal or total external nasal defect and leave a noticeable scar in the flap donor site.

The reliability and versatility of the Converse scalping forehead flap can be explained in terms of the angiosome concept. In 1987 Taylor and Palmer introduced the concept of angiosome as three-dimensional anatomical blocks of tissue supplied by source vessels. ${ }^{8}$ Houseman and Taylor applied this knowledge to explore the territories of the superficial temporal and ophthalmic arteries and to explain the differences in scalp and forehead flaps in regards to vascularity. ${ }^{9}$ They showed that during design of a flap, an adjacent angiosome can safely be incorporated and that enhances the length:breath ratio safely and successfully; but perfusion difficulties may arise when more successive angiosomes are being 
included. The Converse scalping flap is based primarily on the superficial temporal vessels on contralateral side, inviolates the ophthalmic territory on the same side and links with the contralateral ophthalmic and superficial temporal territory to produce a two-territory flap. The galea is supplied from the periphery by deep branches from these vessels along with perforators.

The scalping forehead flap thus has several advantages for reconstruction of large external nasal defects. The 'like' tissue is replaced by the 'like'; forehead skin has properties similar to nasal skin in terms of colour, texture and thickness. The flap has a robust and reliable vascularity and venous drainage. The soft tissue for reconstruction is harvested from the lateral portion of the forehead; thus making the scar less noticeable. The donor-site defect is covered with a full thickness skin graft harvested from the postauricular or supraclavicular region which gives a good colour and texture match. (Fig. 2) Most of the incisions are well hidden within the hair-bearing scalp and once the pedicle of the flap is divided in second stage, the whole hair-bearing scalp is returned to its original position. A large surface area of the flap is available to reconstruct total external nasal defect. The scalping forehead flap has also been used to reconstruct cheeks, eyebrows and orbital roofs. ${ }^{10}$

\section{Conclusion}

The scalping forehead flap is one of the best techniques for total and subtotal nasal reconstruction because this flap is extremely reliable and provides supple skin of good quality with excellent colour and texture match. Even in the era of microvascular free tissue transfer the Converse scalping forehead flap is still an excellent option in the armamentarium of plastic surgeons for subtotal and total nasal reconstruction.

\section{References}

1. Converse JM. New forehead flap for nasal reconstruction. Proc R Soc Med. 1942; 35: 811

2. Converse JM, McCarthy JG. The scalping forehead flap revisited. Clin Plast Surg. 1981; 8: 413

3. Converse JM. Clinical applications of the scalping flap in reconstruction of the nose. Plast Reconstr Surg. 1969; 43: 247

4. Antonyshyn O, Gruss J, Zuker R, Mackinnon SE. Tissue expansion in the head and neck. Sixty-Fifth Annual Meeting of the American Association of Plastic Surgeons. Washington, DC: AAPS, 1986

5. Nichter LS, Morgan RF, Nichter MA. The impact of Indian methods for total reconstruction. Clin Plast Surg. 1983;10:635

6. Tardy ME, Sykes J, Kron T. The precise midline forehead flap in reconstruction of the nose. Clin Plast Surg. 1985;12:481

7. Burget GC, Menick FJ. Aesthetic Reconstruction of the Nose. St Louis: Mosby, 1994: 57-91

8. Taylor GI, Palmer JH. The vascular territories (angiosomes) of the body: experimental study and clinical applications. Br J Plast Surg. 1987; 40:113

9. Houseman ND, Taylor GI, Pan W-R. The angiosomes of the head and neck: anatomic study and clinical applications. Plast Reconstr Surg. 2000;105:2287

10. Converse JM. Full thickness loss of nasal tissue. In Converse JM (Ed.). Reconstructive Plastic Surgery. Vol 2, 2nd Edition. Philadelphia, USA; Saunders; 1977. 